\title{
Self Assembled Phenylene Vinylene Materials
}

\author{
Martin U. Pralle*, Gregory N. Tew, ${ }^{*}$ Mehmet Sayar, ${ }^{* *}$ Leiming Li, ${ }^{* * *}$, and Samuel I. \\ Stupp*** \\ * Ion Optics, Inc., Waltham, MA \\ ** Department of Polymer Science and Engineering, University of Massachusetts at \\ Amherst, Amherst, MA \\ *** Departments of Materials Science and Engineering, Chemistry and Medical School, \\ Northwestern University, Evanston, IL
}

A new class of self assembling rodcoil molecules was recently synthesized and their solid state structure was rigorously characterized. These molecules have a triblock architecture with a rigid rod molecular compound coupled to a diblock coil composed of an oligomeric flexible spacer and a bulky coil. The molecular structure has integrated photonic properties resulting from phenylene vinylene segments in the rigid backbone of the rod segment. It has been found that these molecules aggregate spontaneously when cast from solution into discrete objects with dimensions on the order of a few nanometers. The driving force for this aggregation is the crystallization of rod segments observed by electron diffraction. The aggregates were imaged by transmission electron microscopy using the difference in scattering intensity of the crystallized rod segments and the amorphous coils to generate contrast. From this data we suggest that these aggregate are mushroom like with the rods arranged into a crystalline stem and coils randomly splaying to form the cap. These supramolecular building blocks are packed into nanosheets which stack on top of one another to form macroscopic materials as observed by small angle $x$-ray scattering and TEM.

Detailed cross sectional electron microscopy together with selective staining techniques has documented the nature of the $\mathrm{z}$ direction packing. The mushroom aggregates pack in a parallel rather than anti-parallel arrangement within each layer and furthermore these layers stack in a polar fashion. To probe the structure-property relationship piezoelectric measurements were carried out on these self assembled films and spontaneous piezoelectric activity was demonstrated without prior poling procedures. Upon poling the net polarization was enhanced and piezoelectric activities equal that of quartz were observed. By applying both positive and negative fields a hysteresis was observed suggesting that these materials exhibit ferroelectric character.

Through novel synthesis a supramolecular diode structure was assembled in this way with emissive phenylene vinylene in the rod segment and hole transporting triphenylamine (TPA) groups in the coil. This molecular architecture afforded strong energy transfer as can be seen in figure 1. The TPA absorbs strongly at $302 \mathrm{~nm}$ but then transfers the energy to the phenylene vinylene segment from which light is emitted at visible wavelengths. Through subsequent characterization it was shown that the formation of nanoaggregates is governed by the molecular architecture rather than the specific chemical functions of the 3 molecular segments. 


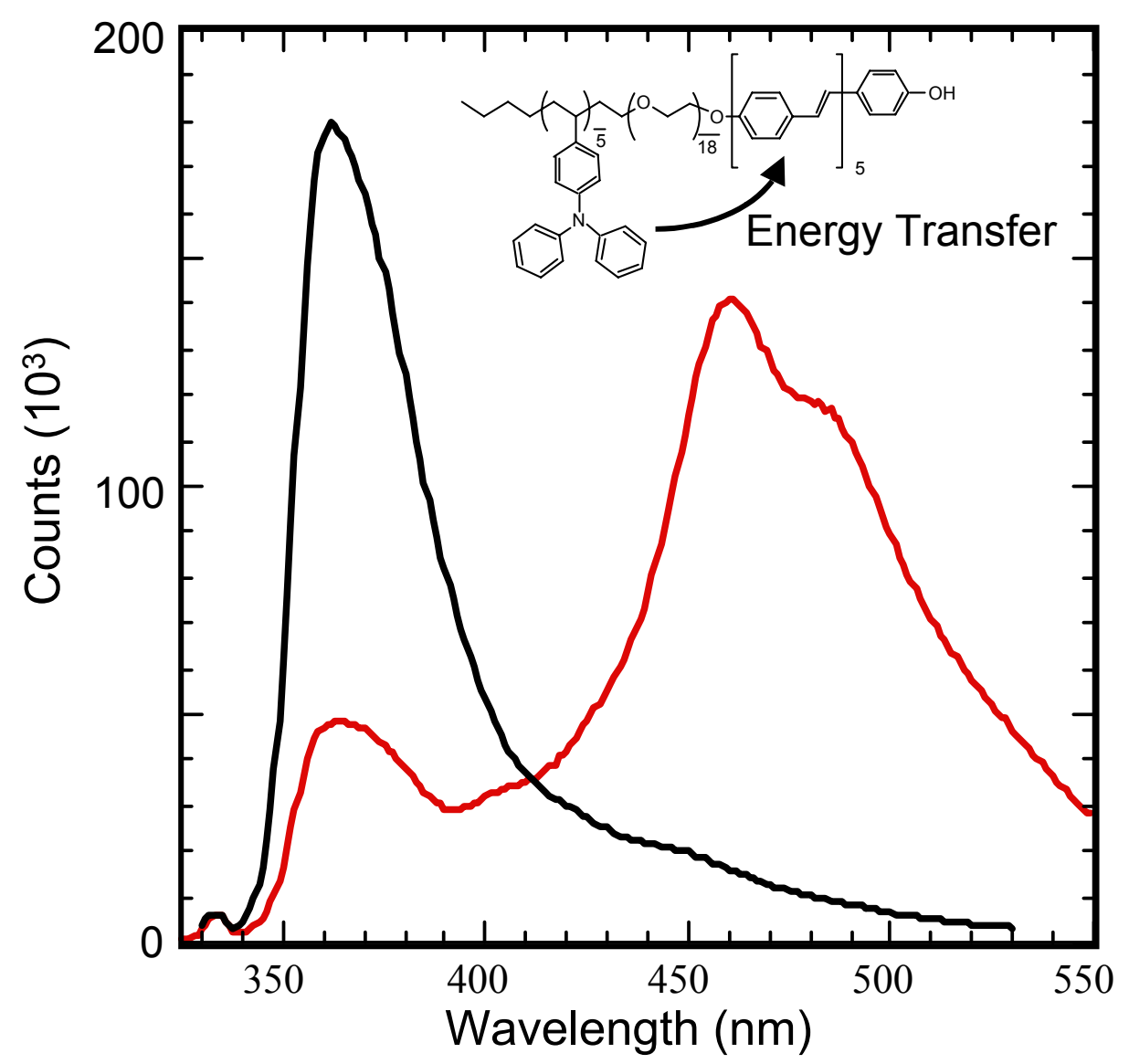

Figure 1. Fluorescence spectra of the TPA coil alone (black) and of the rodcoil molecule (grey) showing a drastic reduction in the TPA fluorescence intensity. The samples were excited at $302 \mathrm{~nm}$ where the phenylene vinylene is nearly transparent and TPA has an absorption maximum. 